\title{
Inhibition of RFX6 Suppresses Invasive Ability of Tumor Cells and Activates T Cell Immune Response Though Notch Pathway in Hepatocellular Carcinoma
}

\section{Mu Song}

Xinjiang Medical University Affiliated Second Hospital

Mulati Kuerban

Xinjiang Medical University

\section{Lu Zhao}

Xinjiang Medical University Affiliated Second Hospital

Xiaolin Peng

Southern Medical University Affiliated Nanhai Hospital

Youqin Xu ( $\sim$ tvxq@smu.edu.cn )

Southern Medical University https://orcid.org/0000-0001-6085-8736

\section{Research}

Keywords: tumorigenesis, Notch, T cells, miRNA, hepatocellular carcinoma

Posted Date: November 8th, 2021

DOI: https://doi.org/10.21203/rs.3.rs-978785/v1

License: (c) (1) This work is licensed under a Creative Commons Attribution 4.0 International License.

Read Full License 


\section{Abstract \\ Background}

DNA-binding protein RFX6 was overexpressed in hepatocellular carcinoma and its expression level was correlated with the prognosis and immune cell infiltration in liver hepatocellular carcinoma. However, the mechanism of abnormal expression and biological effects of RFX6 in liver cancer remains unknown.

\section{Methods}

To understand the specific expressing mechanism of RFX6 in liver cancer, we performed bioinformatic prediction, CHIP-qPCR assay, Co-IP and dual-luciferase assay to assess the regulating mechanism of RFX6. In the meantime, a series of biological experiments in vivo and vitro were conducted to analyze the biological significance of RFX6 in hepatocellular carcinoma.

\section{Results}

We demonstrated that the knockdown of RFX6 in liver cancer cells significantly suppressed the proliferation, migration and invasion of cancer cells. Moreover, inhibition of RFX6 could stimulate immune response of T cells. Among lots of interacting proteins, we revealed RFX6 directly binding to DTX2, regulator of Notch signaling pathway by targeting NOTCH1, and help it`s transcription stability. Furthermore, we discovered miRNA-542-3p, expression of which was decreased in hepatocellular carcinoma, was directly involved in negative regulation of the expression of RFX6.

\section{Conclusions}

In summary, we discovered a miRNA-542-3p-RFX6-DTX2-NOTCH1 regulatory pathway played significant roles in the tumor progression of liver hepatocellular carcinoma.

\section{Background}

Liver cancer is the third leading cause of cancer death worldwide. Although many signaling pathways have been revealed as key mechanism regulating the development of liver cancer and great progress has been made in the tumor treatment, the uncontrollable progression and poor prognosis still result in high mortality. Among all types of liver cancer, hepatocellular carcinoma (HCC) is the most common type comprising $75 \%-85 \%$ of all cases. Hepatocellular carcinoma occurs often in men with age 50 or older and mostly caused by long-term damage of the liver, such as hepatitis B or hepatitis $C$ virus infection, alcohol abuse and autoimmune diseases of liver.[1] Pathogenesis study of HCC involves genome, transcriptome, proteome and metabolism. A variety of key signaling pathways contribute HCC tumorigenesis including MEK/ERK[2], mitogen-activated protein kinases (MAPK)[3], mammalian target of rapamycin (mTOR)[4], 
JAK/STAT[5], Hedgehog[6] and TGF- $\beta$ signaling pathways[7]. Studies have sought to target key components in molecular and cellular pathways that are known to have aberrant signaling in HCC. While numerous targeted agents have been tested, the majority of these agents have failed to produce a survival benefit in clinical trial[8]. Once HCC progresses, limited systemic treatment options are available. The resultant inflammation in combination with altered signaling pathways leads to HCC development[9].

Notch signaling pathway is a highly conserved signaling pathway regulating a series of cell functions, including proliferation[10], apoptosis, invasion, metastasis[11] and differentiation[12]. The Notch system consists of four transmembrane receptors (notch-1, $-2,-3,-4)$ and two types of ligands: the Jagged family ligand (Jagged1, Jagged2) and the Delta family ligand (DLL-1, -3, -4)[13]. Notch1, Notch3, and Notch4 are commonly overexpressed in $\mathrm{HCC}$ and activated Notch signaling pathway has also been shown to promote liver tumor formation in mouse models[14]. Current study have shown Notch1 help promote HBV $X$ protein-induced hepatocellular carcinoma through the Wnt/ $\beta$-catenin pathway[15]. Upregulation of Notch1 help increase the carcinogenesis potential of human HCC cells[16, 17]. Notch1 expression was increased in HCC and tumor metastasis (TNM) stage was significantly associated with increased Notch1 mRNA levels[18]. HCC patients with TNM STAGE III - IV and tumor vein invasion had higher levels of Notch1 expression compared with patients with TNM stage I - II and patients without tumor vein invasion[19]. Knocking out of NOTCH1 eliminated Snail1 expression and effectively inhibited the migration of cancer cells in vitro and in lung metastasis[17]. Matrix metalloproteinase-2 (MMP-2), metalloproteinase-9 (MMP-9) and vascular endothelial growth factor (VEGF) are key factors in tumor invasion and metastasis during angiogenesis[16]. Notch signaling pathway down-regulates MMP-2, MMP-9 and VEGF[12]. In addition, inhibition of Notch1 has been shown to prevent HCC metastasis in vitro and in vivo[20]. Overexpression of Sox9, a Notch pathway regulating gene, was frequently detected in HCC patients and associated with poor prognosis[14]. Furthermore, CD24 promotes HCC progression via triggering Notch-related EMT and modulation of tumor microenvironment[11]. Therefore, the abnormal expression of Notch signal is closely related to the occurrence of hepatocellular carcinoma. Notch has been revealed as a potential therapeutic target for HCC due to its involvement in the proliferation, invasion and metastasis of HCC[21].

RFX6 is an important protein involved in beta-cell maturation and function in pancreas[22]. TCGA data shown its high expression in HCC and was negatively correlated with the prognosis of HCC patients. DTX2, which was predicted as a possible interactive protein of RFX6, is a regulator of Notch signaling[23]. Our study demonstrated a microRNA promoted the mechanism of HCC invasion, metastasis and immune escape by regulating target genes and activating Notch pathway. MiRNA-542-3p has been reported as a potential microRNA inhibiting HCC clinical development but its regulatory mechanism is not clear. Here, we found miRNA-542-3p regulates RFX6 raising the expression of DTX2 resulting in stable expression Notch1 and activating Notch pathway, which major affected in the invasion and immune responses in HCC.

\section{Methods}




\section{Human Cancer Cell Xenograft Model}

All animal work was approved by Institutional Animal Care and Use Committee (IACUC) of Southern Medical University. $3 \times 10^{6}$ liver cancer cells were implanted into the skeletal muscle of the hind limbs of 3 4-week old BALB/c nude mice (nu/nu). One week after transplantation, the diameter of tumors were measured every three days. Tumors were recovered and weighted after three weeks.

\section{Cell Culture}

Human hepatocellular liver carcinoma cell lines (HepG2, Huh7) were purchased from American Type Culture Collection (ATCC, Manassas, VA, USA). Normal liver cell LO2 was purchased from American Type Culture Collection (ATCC, Manassas, VA, USA) and cultured in Roswell Park Memorial Institute 1640 medium (Gibco, USA) supplemented with 10\% fetal bovine serum (FBS, HyClone, Utah, USA) and 1\% Penicillin-Streptomycin (Thermo). HepG2 and Huh7 were cultured in Dulbecco`s Modified Eagle Medium (DMEM) medium (Gibco, USA) supplemented with $10 \%$ fetal bovine serum (FBS, HyClone, Utah, USA) and $1 \%$ Penicillin-Streptomycin (Pen/Strep) (Gibco) at $37^{\circ} \mathrm{C}$ with $5 \% \mathrm{CO}_{2}$.

\section{Establishment of Transfected Cell Lines}

The vectors expressing RFX6-specific siRNA (RIBOBIO, Cat\# SI00701841, Cat\# SI04193749, Cat\# SI04249056), DTX2-specific siRNA (RIBOBIO, Cat\# SI04728740,). Vector expressing human RFX6 cDNA and DTX2 cDNA were transfected into cells. Cell were selected with puromycin $(2 \mu \mathrm{g} / \mathrm{ml}$, GeneChem) for three days for stable transfection. Details of siRNA sequence were listed as follows.

RFX6 siRNA-1: TACGCTCATAATGATGTACAA

RFX6 siRNA-2: AAGCCGAGGAAGTGTCATTAA

RFX6 siRNA-3: CAGCGACGCTGTGAAGAATGA

DTX2 siRNA: GAGTGTTCTGATGTCAGCCATTGGA

\section{Western Blot Analysis}

Cells were extracted for total proteins using lysis buffer and samples were separated on 8-15\% SDS PAGE and transferred to nitrocellulose membranes, which were blocked with blocking buffer ( $5 \%$ skim milk in PBS with $0.05 \%$ Tween 20 ) and incubated with primary antibody in the blocking buffer. After being washed three times with blocking buffer, the membrane was probed with secondary antibody and developed with SuperSignal West Pico (Thermo Fisher Scientific).

\section{Quantitative PCR Analysis}

Real time PCR analysis was performed using StepOnePlus Real-Time PCR System (Applied Biosystems) with FastStart Universal SYBR Green Master (Roche) as previously reported. The primer sets used were as 
follows,

GAPDH-F: GAACGGGAAGCTCACTGG;

GAPDH-R: GCCTGCTTCACCACCTTCT;

RFX6-F: GGTACCATGGCCAAGGTCCCGGAGCTG;

RFX6-R: TCTAGATTAAGTGCCTCCAGCTGCTGTTC;

DTX2-F: GGTACCATGGCCATGGCCCCAAGCCC;

DTX2-R: GGTACCTCACTGCTGCTCCAGGCAGTCC;

U6-F: GCTTCGGCAGCACATATACTAAAAT;

U6-R: CGCTTCATGAATTTGCGTGTCAT;

miRNA-542-3p: TGTGACAGATTGATAACTG;

miR-542-3p-RFX6 mut-F: TGATTTGACAGTGTTAGCAGCATTCCGATTCTATG;

miR-542-3p-RFX6 mut-F-R: CTGCTAACACTGTCAAATCATCACACTAATGCAC;

The PCR conditions were as follows: $10 \mathrm{~min}$ at $95^{\circ} \mathrm{C}, 40$ cycles of $15 \mathrm{~s}$ at $95^{\circ} \mathrm{C}$, and $1 \mathrm{~min}$ at $60^{\circ} \mathrm{C}$. The average $\mathrm{Ct}$ value for each gene was determined from triplicate reactions and normalized with the amount of GAPDH for gene and U6 for miRNA.

\section{Cell Proliferation, Apoptosis, Migration Assay and Invasion Assay}

Cells transfected with various plasmids were seeded onto 96-well plate (Corning Inc, Corning, NY, USA) at a density of $1 \times 10^{4}$ cells/ well in 96-well plates. At different time points ( $24 \mathrm{~h}, 48 \mathrm{~h}$ and $72 \mathrm{~h}$ ) after plating, the number of cells was assessed using cell counting kit 8 according to the manufacturer's protocol (Dojindo, Tokyo, Japan). The transfected cell lines undergoing apoptosis were distinguished from live and necrotic cells by using Annexin-V and Propidium iodide (PI) staining Kit (Calbiochem, San Diego, CA, USA). All experiments were independently repeated for three times.

For cell invasion assay, $1.5 \times 10^{5}$ cells in serum-free medium were seeded into a matrigel coated chamber ( $8 \mu \mathrm{m}$ pore size, Corning Incorporated, NY, USA) and the lower chamber was immediately filled with $500 \mu \mathrm{l}$ of 1640 medium with $10 \%$ FBS as a chemoattractant. After $24 \mathrm{~h}$ of incubation, the non-invading cells were removed from the upper chamber by a cotton swab and the membranes fixed with methanol and stained by $0.1 \%$ crystal violet. The Data were represented as mean \pm standard deviation $(\mathrm{sd}), \mathrm{n}=3$.

\section{Dual-luciferase Reporter Assay}


Cells were seeded in triplicate onto 6-well plates at a density of $4 \times 10^{5}$ cells/well for $48 \mathrm{~h}$ and transfected with $0.3 \mu \mathrm{g}$ of certain plasmids separately together with $30 \mathrm{ng}$ of pGMR TK renilla plasmid (GenomeDitech, Shanghai, China) using Lipofectamine ${ }^{\mathrm{TM}} 3000$ reagent (Invitrogen, Carlsbad, USA). Luciferase and renilla activities were measured using the Dual Luciferase Reporter Assay Kit (Promega, Madison, USA) after $48 \mathrm{~h}$ transfection.

\section{Quantitative Real-Time PCR for Chromatin Immunoprecipitation Analysis (CHIP-qPCR Assay)}

Formaldehyde was directly added into the cell culture medium, after shaking and fixation for 10 minutes. Glycine was quickly added into mixture for 5 minutes and then cells were washed with PBS and scraped off into ep tubes. Specific antibody, IgG and magnetic beads (or protein $A / G$ ) that bind antibodies were added into ultrasonic centrifugal products as mentioned above. The ultrasonic products were divided into two parts, specific antibody and IgG were added and were rotated overnight. Magnetic beads were added and rotated for $4 \mathrm{~h}$. Washed with dilute buffer solution, low salt solution, high salt solution, lithium chloride solution and TE buffer solution for 7 times. Put the products into eluting buffer at $65^{\circ} \mathrm{C}$ overnight. The elution products were purified with DNA purification kit prepared for ChIP detection. Primers were designed according to the range of 2000-3000bp upstream of the ORF region of the prediction gene in stages. Samples were screened by quantitative PCR with primers. The bound primers screened by PCR were verified by QPCR with Input and IP samples. Sequence of ChIP-qPCR were listed as follows, F: AATGAGTGAGCAGGCGAAGG, ChIP-R: GAAATGTAGTCCCGGTAGGGC.

\section{Co-immunoprecipitation Assay}

Cells were lysed in RIPA duffer containing protease and phosphatase inhibitors and cells were collected after $12,000 \times \mathrm{g}$ for $10 \mathrm{~min}$ at $4^{\circ} \mathrm{C}$ centrifugation for immunoprecipitation assays. Supernatants were immunoprecipitated with antibodies followed by incubation with magnetic protein $A / G$ beads (Pierce) for $2 \mathrm{~h}$ at $4^{\circ} \mathrm{C}$. The immune complexes were washed three times with PBS duffer, re-suspended in SDS-PAGE buffer and analyzed by Western blot analysis.

\section{Statistical Analysis}

Data were analyzed using SPSS 20.0. and two-tailed independent Student`s t-test, $P<0.05$ was considered significant. Two patient cohorts were compared by Kaplan-Meier survival plot and log rank $\mathrm{P}$ values are calculated.

\section{Results}

RFX6 highly expressed in hepatocellular carcinoma and negatively correlated with the prognosis.

The RNA-seq data of liver hepatocellular carcinoma (LIHC) and paired adjacent normal tissues in TCGA database (https://portal.gdc.cancer.gov/) shown RFX6 was overexpressed in hepatocellular carcinoma (Fig. 1a). Positive RFX6 expression level was closely correlated with histologic grade (Fig. 1b), pathologic 
stage and TNM stage (Fig. 1c). The relative protein expression of RFX6 in hepatocellular carcinoma tissues was higher than that in adjacent normal tissues (Fig. 1d). In the meantime, we examined the expression levels of RFX6 mRNA and protein in HepG2, Huh7 and LO2 cell lines. Results shown in Fig. S1a and S1b, the expression levels of RFX6 mRNA and protein were increased in HepG2 and Huh7 when compared to normal liver cell LO2.

According to RFX6 expression value, the samples were divided into high and low expression group (median division), and the difference between the two groups was calculated. GSEA (Gene Set Enrichment Analysis) revealed RFX6 may positively related to TH17 cell differentiation, B cell and T cell receptor signaling pathway (Fig. 2a). KEGG analysis indicated that RFX6 possibly involved in multiple pathways especially in EMT pathways in cancer including NF-kB and JAK-STAT pathway (Fig. 2b). Due to the possible role of RFX6 in tumor immune response, we estimated the immune infiltration with CIBERSORT [24]. The correlation between RFX6 expression and immune infiltration of each immune cell type was shown in bubble diagram. It's worth noting that RFX6 was negatively related to T cell activation (Fig. 2c).

\section{Expression of RFX6 promotes tumorigenesis and T cell immune response of hepatocellular carcinoma.}

The high expression of RFX6 in HCC and its negative role in prognosis and CD8+ T cells infiltration support the notion that RFX6 help promotes liver hepatocellular tumorigenesis and may related to antitumor immunity mediated by CD8+ T cells. In order to investigate the roles of RFX6 in HCC, we regulated the expression level of RFX6 in HepG2 and Huh7 by knockdown and overexpression Fig. S1c and S1d. Downregulating RFX6 decreased the cellular proliferation and survival of HepG2 and Huh7, while increased the expression of RFX6 in cells could stimulated the proliferation and inhibited cell apoptosis. (Fig. 3a-b and Fig. S2a-b). Transwell assays indicating the expression of RFX6 significantly promoted the migration and invasion abilities of HepG2 and Huh7 (Fig. 3c-d and Fig. S2c-d). Knockdown of RFX6, by contrast, got the opposite results of suppression of tumor cell migration and invasion (Fig. 3c-d and Fig. S2c-d). Furthermore, the suppression of RFX6 expression in HepG2 cells significantly reduced the growth of xenograft formed by HepG2 cells in immunodeficient mice (Fig. 3e).

As results shown in Fig. 2c-d, RFX6 may be relevant with the activation of T cell in HCC. Therefore, we detected 23 known marker genes of different immune cell types by qPCR under different RFX6 expression levels in HepG2 and Huh7. Noting that positive RFX6 expression was relevant with regulatory T cells and inversely correlated with dysfunction of progressive $T$ cell by affecting receptors of effector $T$ cell such as CTLA-4, PD-1and LAG3 (Fig. 4a-b). The list of 23 markers were shown in Table 1. These results indicating that RFX6 expression induced progressive $T$ cell exhaustion. 
Table 1

23 marker genes regarding to tumor immunity.

\begin{tabular}{|ll|}
\hline Primer Name & 5' sequence 3 \\
\hline Has-TRIB3-F & CTAGGACCACCCTACTACAC \\
\hline Has-TRIB3-R & CACCTGATAAGCACCCAAGC \\
\hline Has-CTLA4-F & GACAGAGCTGGGATGTTTCTG \\
\hline Has-CTLA4-R & CGGCTATAAACGTCTCATACG \\
\hline Has-PD1-F & GTGAGCATGAAACTATGCACC \\
\hline Has-PD1-R & GCCACTTAAGGAACCAGTGC \\
\hline Has- LAG3-F & GCTTCAACGTCTCCATCATG \\
\hline Has- LAG3-R & GGCTCACATCCTCTAGTCG \\
\hline Has-TIM3-F & GGCATCTACATCGGAGCAG \\
\hline Has-TIM3-R & GTGGTTGGATCTATGGCATTG \\
\hline Has-IFNY-F & CGGTAACTGACTTGAATGTCC \\
\hline Has-IFNY-R & CAGGCAGGACAACCATTAC \\
\hline Has-IL2-F & CTCACAGTAACCTCAACTCC \\
\hline Has-IL2-R & CCTCCAGAGGTTTGAGTTC \\
\hline Has-FOXP3-F & GAAGGTCTTCGAAGAGCCAG \\
\hline Has-FOXP3-R & GTCGGATGATGCCACAGATG \\
\hline Has-TNFa-F & GGTATGAGCCCATCTATCTGG \\
\hline Has-TNFa-R & CAGAAGAGGTTGAGGGTGTC \\
\hline Has-TGFß-F & GCAAGACTATCGACATGGAG \\
\hline Has-TGFß-R & GGTTTCCACCATTAGCACG \\
\hline Has-IL6-F & GTAGTGAGGAACAAGCCAGAG \\
\hline Has-IL6-R & GCAGGAACTCCTTAAAGCTG \\
\hline Has-IL10-R & CACCCTGATGTCTCAGTTTCG \\
\hline CTGATGGGAACGTGGACTAC \\
\hline GCCAAGTGTTACCTCTGAAGC \\
\hline HasTTCCACAGGCACAAGC \\
\hline HACCAAGACCCAGACATCAAG
\end{tabular}




\begin{tabular}{|ll|}
\hline Primer Name & $\mathbf{5}$ ' sequence $\mathbf{3}$ \\
\hline Has-IL4-F & CGTACTCTGGTTGGCTTCC \\
\hline Has-CD3-F & CTTCACACACAGACTGTTGTC \\
\hline Has-CD3-R & CTAGCATCTGCGCTTTCTC \\
Has-CD80-F & CTTCAGAGACTATCTGATTTCC \\
\hline Has-CD80-R & GACTACTGCTTTGACGTACC \\
\hline Has-CD86-F & CAAGCCATAGTGGAGAGAAC \\
\hline Has-CD86-R & CTGCTGTCTGTCTTATGTCC \\
\hline Has-CD68-F & CCATCTTGCTGCCTCTCATC \\
\hline Has-CD68-R & GTCTTTGAGCCAGTTGCGTG \\
\hline Has-CD206-F & GTAATGCATTTGCGTGGCTG \\
\hline Has-CD206-R & GCAATGTGCTGTCTTCCAG \\
\hline Has-CD163-F & GACTCTTGGGACTTGGACG \\
\hline Has-CD163-R & CCACAAGGAAGACTCATTCC \\
\hline Has-LEF1-F & CTAATGCACGTGAAGCCTCAGC \\
\hline Has-LEF1-R & GTCTCTTGCAGACCAGCCTG \\
\hline Has-TCF7-F & GATCTCATGGAAACTGGCCAG \\
\hline Has-TCF7-R & GCTGGCAAGACAGATGGTAC \\
\hline Has-GZMB-F & GCTTCCTGATACGAGACGAC \\
\hline Has-GZMB-R & CGATCTTCCTGCACTGTCATC \\
\hline
\end{tabular}

miRNA-542-3p negatively regulates RFX6 and positively correlates with the prognosis of human hepatocellular carcinoma.

Considering the importance of RFX6 in promoting liver cell tumorigenesis and worthy role of miRNA in tumor suppression and oncogenesis, we used miRDB prediction website (http://mirdb.org/) to predict miRNAs that might target RFX6. According to the expression level in liver hepatocellular carcinoma and target score, 36 miRNAs (list shown in Table 2) were selected as potential regulators of RFX6 mRNA. By analysis of TCGA datasets, we found only one candidate -miRNA-542-3p was highly expressed in tumor adjacent normal tissues, comparing to liver hepatocellular carcinoma tissues (Fig. 5a). Consistent result was found in hepatocellular carcinoma cell line HepG2 and Huh7 (Fig. 5b). Moreover, the expression level of miRNA-542-3p was negatively related with the prognosis of HCC patients (Fig. $5 c$ ). To determine the impact of miRNA-542-3p on the expression of RFX6, we used miRNA mimics and inhibitor to demonstrate 
the expression levels of miRNA-542-3p was inversely correlated with the expression level of RFX6

(Fig. 5d). Dual-luciferase reporter assay detected that mutating the predicted sites of miRNA-542-3p in the 3'UTR of RFX6 mRNA significantly heightened the fluorescence ratio (Fig. 5e). These data confirmed that miRNA-542-3p regulated RFX6 in hepatocellular carcinoma cells. 
Table 2

miRNA prediction results of RFX6 mRNA

\begin{tabular}{|c|c|c|c|c|}
\hline Target Rank & Target Score & miRNA Name & Gene Symbol & Gene Description \\
\hline 1 & 99 & hsa-miR-30b-5p & RFX6 & regulatory factor $\mathrm{X} 6$ \\
\hline 2 & 99 & hsa-miR-30a-5p & RFX6 & regulatory factor $\mathrm{X} 6$ \\
\hline 3 & 99 & hsa-miR-30c-5p & RFX6 & regulatory factor $X 6$ \\
\hline 4 & 99 & hsa-miR-30d-5p & RFX6 & regulatory factor $\mathrm{X} 6$ \\
\hline 5 & 99 & hsa-miR-30e-5p & RFX6 & regulatory factor $X 6$ \\
\hline 6 & 97 & hsa-miR-935 & RFX6 & regulatory factor $\mathrm{X} 6$ \\
\hline 7 & 94 & hsa-miR-376c-3p & RFX6 & regulatory factor $\mathrm{X} 6$ \\
\hline 8 & 91 & hsa-miR-510-3p & RFX6 & regulatory factor $X 6$ \\
\hline 9 & 88 & hsa-miR-664a-3p & RFX6 & regulatory factor $X 6$ \\
\hline 10 & 85 & hsa-miR-4500 & RFX6 & regulatory factor $X 6$ \\
\hline 11 & 82 & hsa-let-7d-5p & RFX6 & regulatory factor $\mathrm{X} 6$ \\
\hline 12 & 82 & hsa-let-7c-5p & RFX6 & regulatory factor $\mathrm{X} 6$ \\
\hline 13 & 82 & hsa-let-7i-5p & RFX6 & regulatory factor $X 6$ \\
\hline 14 & 82 & hsa-miR-98-5p & RFX6 & regulatory factor $X 6$ \\
\hline 15 & 82 & hsa-let-7a-5p & RFX6 & regulatory factor $X 6$ \\
\hline 16 & 82 & hsa-let-7b-5p & RFX6 & regulatory factor $X 6$ \\
\hline 17 & 82 & hsa-let-7g-5p & RFX6 & regulatory factor $\mathrm{X} 6$ \\
\hline 18 & 82 & hsa-let-7e-5p & RFX6 & regulatory factor $X 6$ \\
\hline 19 & 82 & hsa-miR-4458 & RFX6 & regulatory factor $\mathrm{X} 6$ \\
\hline 20 & 82 & hsa-let-7f-5p & RFX6 & regulatory factor $X 6$ \\
\hline 21 & 81 & hsa-miR-548an & RFX6 & regulatory factor $X 6$ \\
\hline 22 & 81 & hsa-miR-153-5p & RFX6 & regulatory factor $X 6$ \\
\hline 23 & 77 & hsa-miR-4501 & RFX6 & regulatory factor $X 6$ \\
\hline 24 & 77 & hsa-miR-648 & RFX6 & regulatory factor $X 6$ \\
\hline 25 & 76 & hsa-miR-587 & RFX6 & regulatory factor $\mathrm{X} 6$ \\
\hline 26 & 76 & hsa-miR-3194-3p & RFX6 & regulatory factor $\mathrm{X} 6$ \\
\hline 27 & 75 & hsa-miR-542-3p & RFX6 & regulatory factor $X 6$ \\
\hline
\end{tabular}




\begin{tabular}{|lllll|}
\hline Target Rank & Target Score & miRNA Name & Gene Symbol & Gene Description \\
\hline 28 & 75 & hsa-miR-9-5p & RFX6 & regulatory factor X6 \\
\hline 29 & 75 & hsa-miR-548m & RFX6 & regulatory factor X6 \\
\hline 30 & 75 & hsa-miR-6514-5p & RFX6 & regulatory factor X6 \\
\hline 31 & 75 & hsa-miR-653-3p & RFX6 & regulatory factor X6 \\
\hline 32 & 75 & hsa-miR-10523-5p & RFX6 & regulatory factor X6 \\
\hline 33 & 72 & hsa-miR-4699-3p & RFX6 & regulatory factor X6 \\
\hline 34 & 71 & hsa-miR-506-3p & RFX6 & regulatory factor X6 \\
\hline 35 & 71 & hsa-miR-124-3p & RFX6 & regulatory factor X6 \\
\hline 36 & 70 & hsa-miR-203a-3p & RFX6 & regulatory factor X6 \\
\hline
\end{tabular}

\section{Downregulation of RFX6 by miRNA-542-3p affects invasion and oncogenesis of hepatocellular carcinoma cells.}

Since miRNA-542-3p suppresses RFX6 expression in hepatocellular carcinoma cells, we wonder if such regulation affects the tumorigenesis of liver cells. To investigate the role of miRNA-542-3p, we induced miRNA-542-3p expression by mimics in HepG2 and Huh7 cells. Proliferation, migration and invasion of HCC cells were significantly inhibited by the overexpression of miRNA-542-3p (Fig. 6a-C). Moreover, progressive $T$ cell were notably inhibited by the expression of miRNA-542-3p (Fig. S3 a). Consistently, the overexpression of miRNA-542-3p could suppress the tumorigenesis induced by RFX6 overexpression (Fig. 6d-g and Fig. S3 b-c). Therefore, these results indicate that miRNA-542-3p could inhibit HCC tumorigenesis by suppressing the expression of RFX6.

RFX6 contributes to invasion and immune escape through Notch pathway by promoting the translation of DTX2 in hepatocellular carcinoma.

As a transcription factor, we predicted RFX6 probably functioned by regulating the stability of mRNAs relating to tumorigenesis in HCC. For purpose of understanding the mechanism of RFX6 promotes HCC tumorigenesis, we used a functional association network database STRING (https://string-db.org/) and UniProt (https://www.uniprot.org/) to predict the targets that might interact with RFX6. The intersection of predicted outcomes was DTX2, a probable E3 ubiquitin-protein ligase (Fig. 7a). DTX2 regulates the Notch pathway via ubiquitin ligase activity in vitro.

Most of the potential targets were involved in Notch pathway (Fig. 7b). As RFX6 was reported as a transcription factor, we used ChIP-qPCR to determine the target gene of RFX6. So that, we confirmed the interaction between RFX6 and DTX2 (Fig. 7c). Meanwhile, the mRNA level of DTX2 was positively correlated with the protein levels of RFX6 (Fig. 7d and Fig. S3 d). These data support the notion that RFX6 help regulate the transcription of DTX2. To determine the interact protein of DTX2 in HCC, we used Co-IP 
assay to identify STRING candidates of Notch pathway. Of all 6 potential proteins, Notch 1 was confirmed to be interacted with DTX2 (Fig. 7e). Furthermore, we used miR-542-3p mimic to suppress the expression of RFX6 and further inhibited the mRNA express level of DTX2 (Fig. S3 e), HCC tumorigenesis level was retrained by downregulation of DTX2 conducted by miR-542-3p (Fig. $7 f$ and Fig. S3 f).

\section{Discussion}

Hepatocellular carcinoma develops predominantly with cirrhosis and hepatitis. The absence of curative treatment methods and rapid drug resistance result in high mortality rate. It is urgent to conduct extensive research towards mechanism of HCC development and decreasing the cancer cell resistance to treatment and immune response. RFX 6 is known as a transcription factor required to endocrine pancreas development and regulates beta-cell maturation and function. Our study discovered that the RFX6 was overexpressed in HCC and its expression predicts poor prognosis of HCC patients, but the role of RFX6 in tumorigenesis remain unclear. By knockdown of RFX6 in HCC cells, we found the absence of RFX6 induced higher cell apoptosis rate and poor proliferation, migration and invasion ability. DTX2 is a regulator of Notch signaling pathway involved in cell fate and tumor immunology. We discovered that RFX6 could promote transcription of DTX2 and was regulated by miRNA-542-3p in HCC, which positively affected the tumorigenesis and inhibited CD8 T cell activation in HCC.

Considering the important roles of RFX6 in tumorigenesis, we investigated the critical regulator of RFX6 by dual-luciferase reporter assay system. Published and our data indicate that miRNA-542-3p are downregulated in different types of human cancers, including breast cancer, hepatocellular carcinoma, lung cancer and so on. These findings suggesting that miRNA-542-3p is involved in tumor suppression. We found miRNA-542-3p is positively correlated with good prognosis in HCC. There is limited information of the regulating mechanism of miRNA-542-3p. Therefore, identify the upstream suppressive pathways that regulates the expression of miRNA-542-3p is a promising research area. Our discovery of the functional link between miRNA-542-3p, RFX6 and DTX2 play s critical roles in hepatocellular carcinoma and provides potential therapeutic targets for hepatocellular carcinoma treatment.

\section{Conclusions}

We discovered a miRNA-542-3p-RFX6-DTX2-NOTCH1 regulatory pathway played significant roles in the tumor progression and provides new potential therapeutic targets for live hepatocellular carcinoma.

\section{List Of Abbreviations}

RFX6, regulatory factor X6; DTX2, deltex E3 ubiquitin ligase 2; KCTD9, potassium channel tetramerization domain containing 9; TRIP6, thyroid hormone receptor interactor 6; NOTCH1, Neurogenic locus notch homolog protein 1; ROC, receiver operator characteristic; GSEA, Gene Set Enrichment Analysis; KEGG, Kyoto Encyclopedia of Genes and Genomes; EMT, Epithelial-Mesenchymal Transition; miRDB, MicroRNA Target Prediction Database; ChIP, Chromatin immunoprecipitation assay. 


\section{Declarations}

\section{Ethics approval and consent to participate}

All institutional and national guidelines for the care and use of laboratory animals were followed.

\section{Consent for publication}

Not applicable.

\section{Availability of data and materials}

The datasets supporting the conclusions of this article are included within the article and its additional files.

\section{Competing interests}

The authors declare that they have no competing interests.

\section{Funding}

This study is supported by Natural Science Foundation of Xinjiang Uygur Autonomous Region (Grant No. 2017D01C249).

\section{Authors' contributions}

MS performed the cell function analysis of different cell lines and Western blot analysis and was a major contributor in writing the manuscript. MK carried out mechanistic research regarding Co-IP and ChIPQPCR, as well as interpreted the prognosis of HCC patients and carried out an in vitro tumorigenesis experiment. LZ was responsible for preprocessing the clinical tissue regarding cancer, adjacent normal tissue and conducted all qPCR analyses in this study. XP established the transfected cell lines and analyzed the miRNA candidate of the target gene. YX confirmed that data/figure presentation accurately reflected the original and oversaw the fellows who participated in this study. All authors read and approved the final manuscript.

\section{Acknowledgements}

Not applicable.

\section{References}

1. Sung H, Ferlay J, Siegel RL, Laversanne M, Soerjomataram I, Jemal A, Bray F. Global Cancer Statistics 2020: GLOBOCAN Estimates of Incidence and Mortality Worldwide for 36 Cancers in 185 Countries. CA Cancer J Clin. 2021;71(3):209-49. 
2. Shah AD, Bouchard MJ, Shieh AC. Interstitial Fluid Flow Increases Hepatocellular Carcinoma Cell Invasion through CXCR4/CXCL12 and MEK/ERK Signaling. PLoS One. 2015;10(11):e0142337.

3. Moon H, Ro SW. MAPK/ERK Signaling Pathway in Hepatocellular Carcinoma. Cancers (Basel) 2021, 13(12).

4. Diniz PHC, Silva SDC, Vidigal PVT, Xavier MAP, Lima CX, Faria LC, Ferrari TCA. Expression of MAPK and PI3K/AKT/mTOR Proteins according to the Chronic Liver Disease Etiology in Hepatocellular Carcinoma. J Oncol. 2020;2020:4609360.

5. Toh TB, Lim JJ, Hooi L, Rashid M, Chow EK. Targeting Jak/Stat pathway as a therapeutic strategy against SP/CD44+ tumorigenic cells in Akt/beta-catenin-driven hepatocellular carcinoma. J Hepatol. 2020;72(1):104-18.

6. Tripathy A, Thakurela S, Sahu MK, Uthansingh K, Singh A, Narayan J, Ajay AK, Singh V, Kumari R. Fatty changes associated with N-Nitrosodiethylamine (DEN) induced hepatocellular carcinoma: a role of sonic hedgehog signaling pathway. Genes Cancer. 2020;11(1-2):66-82.

7. Gonzalez-Sanchez E, Vaquero J, Fernandez-Barrena MG, Lasarte JJ, Avila MA, Sarobe P, Reig M, Calvo M, Fabregat I. The TGF-beta Pathway: A Pharmacological Target in Hepatocellular Carcinoma? Cancers (Basel) 2021, 13(13).

8. Anwanwan D, Singh SK, Singh S, Saikam V, Singh R. Challenges in liver cancer and possible treatment approaches. Biochim Biophys Acta Rev Cancer. 2020;1873(1):188314.

9. Yarchoan M, Agarwal P, Villanueva A, Rao S, Dawson LA, Llovet JM, Finn RS, Groopman JD, El-Serag HB, Monga SP, et al. Recent Developments and Therapeutic Strategies against Hepatocellular Carcinoma. Cancer Res. 2019;79(17):4326-30.

10. Wang R, Sun Q, Wang P, Liu M, Xiong S, Luo J, Huang H, Du Q, Geller DA, Cheng B. Notch and Wnt/beta-catenin signaling pathway play important roles in activating liver cancer stem cells. Oncotarget. 2016;7(5):5754-68.

11. Wan X, Cheng C, Shao Q, Lin Z, Lu S, Chen Y. CD24 promotes HCC progression via triggering Notchrelated EMT and modulation of tumor microenvironment. Tumour Biol. 2016;37(5):6073-84.

12. Caporarello N, Lupo G, Olivieri M, Cristaldi M, Cambria MT, Salmeri M, Anfuso CD. Classical VEGF, Notch and Ang signalling in cancer angiogenesis, alternative approaches and future directions (Review). Mol Med Rep. 2017;16(4):4393-402.

13. Anusewicz D, Orzechowska M, Bednarek AK. Notch Signaling Pathway in Cancer-Review with Bioinformatic Analysis. Cancers (Basel) 2021, 13(4).

14. Chen M, Lu C, Lu H, Zhang J, Qin D, Liu S, Li X, Zhang L. Farnesoid X receptor via Notch1 directs asymmetric cell division of Sox $9(+)$ cells to prevent the development of liver cancer in a mouse model. Stem Cell Res Ther. 2021;12(1):232.

15. Kong F, Hu W, Zhou K, Wei X, Kou Y, You H, Zheng K, Tang R. Hepatitis B virus X protein promotes interleukin-7 receptor expression via NF-kappaB and Notch1 pathway to facilitate proliferation and migration of hepatitis B virus-related hepatoma cells. J Exp Clin Cancer Res. 2016;35(1):172. 
16. Sui C, Zhuang C, Sun D, Yang L, Zhang L, Song L. Notch1 regulates the JNK signaling pathway and increases apoptosis in hepatocellular carcinoma. Oncotarget. 2017;8(28):45837-47.

17. Wang XQ, Zhang W, Lui EL, Zhu Y, Lu P, Yu X, Sun J, Yang S, Poon RT, Fan ST. Notch1-Snail1-Ecadherin pathway in metastatic hepatocellular carcinoma. Int J Cancer. 2012;131(3):E163-72.

18. Wang S, Cai L, Zhang F, Shang X, Xiao R, Zhou H. Inhibition of EZH2 Attenuates Sorafenib Resistance by Targeting NOTCH1 Activation-Dependent Liver Cancer Stem Cells via NOTCH1-Related MicroRNAs in Hepatocellular Carcinoma. Transl Oncol. 2020;13(3):100741.

19. Zhang Y, Zhang Y, Wang J, Gu H. Amarogentin Inhibits Liver Cancer Cell Angiogenesis after Insufficient Radiofrequency Ablation via Affecting Stemness and the p53-Dependent VEGFA/DII4/Notch1 Pathway. Biomed Res Int. 2020;2020:5391058.

20. Wang X, Wang R, Bai S, Xiong S, Li Y, Liu M, Zhao Z, Wang Y, Zhao Y, Chen W, et al. Musashi2 contributes to the maintenance of CD44v6+ liver cancer stem cells via notch1 signaling pathway. $J$ Exp Clin Cancer Res. 2019;38(1):505.

21. Wu G, Wilson G, George J, Qiao L. Modulation of Notch signaling as a therapeutic approach for liver cancer. Curr Gene Ther. 2015;15(2):171-81.

22. Lu J, Cheng C, Cheng ZC, Wu Q, Shen H, Yuan MX, Zhang B, Yang JK. The dual role of RFX6 in directing beta cell development and insulin production. J Mol Endocrinol. 2021;66(2):129-40.

23. Maki K, Sasaki K, Sugita F, Nakamura Y, Mitani K. Acute myeloid leukemia with $t(7 ; 21)(q 11.2 ; q 22)$ expresses a novel, reversed-sequence RUNX1-DTX2 chimera. Int J Hematol. 2012;96(2):268-73.

24. Chen B, Khodadoust MS, Liu CL, Newman AM, Alizadeh AA. Profiling Tumor Infiltrating Immune Cells with CIBERSORT. Methods Mol Biol. 2018;1711:243-59.

\section{Figures}


a
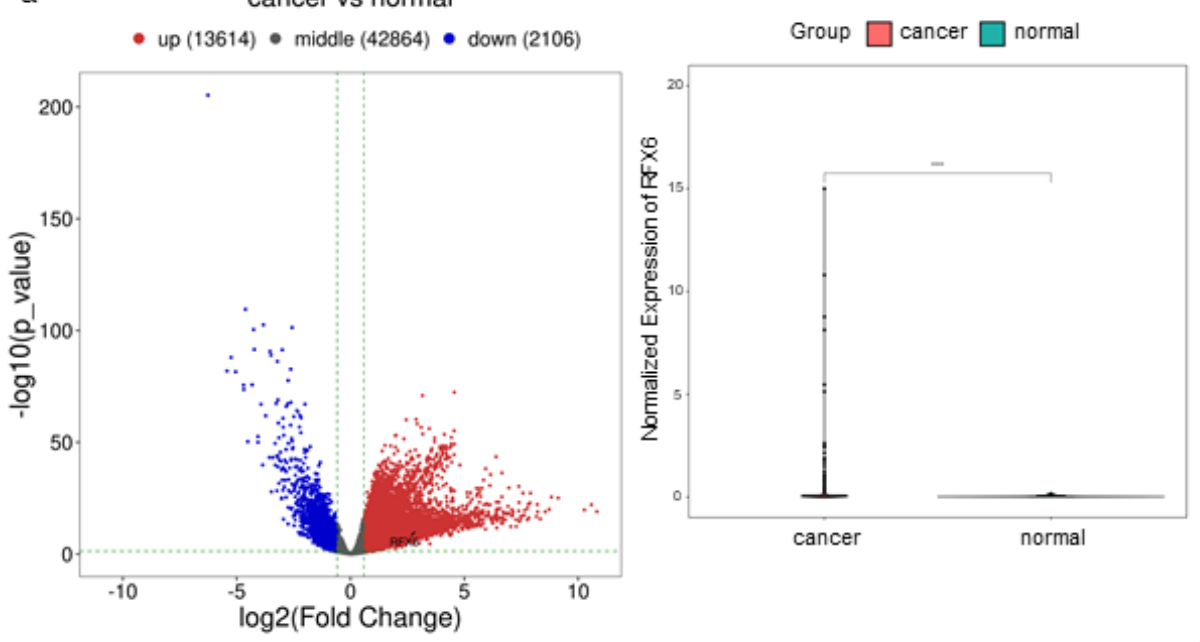

C

\begin{tabular}{|c|c|c|c|c|}
\hline Variable & $\mathrm{HR}$ & $95 \% \mathrm{Cl}$ & P_value & \\
\hline RFX6 & 1.14 & $1.03 \cdot 1.26$ & 0.0139 & - \\
\hline Age & 1.01 & $0.999-1.03$ & 0.0775 & : \\
\hline Pathologic_Stage & 1.66 & $1.36 \cdot 2.03$ & $6.09 \mathrm{e}-07$ & $\rightarrow$ \\
\hline T_Stage & 1.63 & $1.38 \cdot 1.93$ & $1.6 \mathrm{e}-08$ & $\rightarrow$ \\
\hline N_Stage & 1.22 & $1.02-1.47$ & 0.0318 & 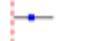 \\
\hline M_Stage & 1.28 & $1.06 \cdot 1.53$ & 0.00983 & $\rightarrow-$ \\
\hline Sex & 0.816 & $0.573 \cdot 1.16$ & 0.26 & \\
\hline Race & 1.08 & $0.959-1.23$ & 0.196 & - \\
\hline Tumor_purity & 1.15 & $0.284 \cdot 4.62$ & 0.848 & \\
\hline
\end{tabular}

b

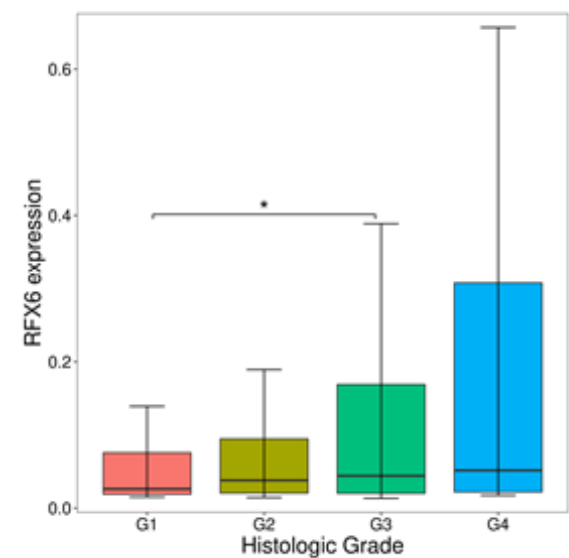

d

RFX6

$\mathrm{HCC} A \mathrm{AD} \mathrm{HCC} \quad \mathrm{AD} \quad \mathrm{HCC} \quad \mathrm{AD}$

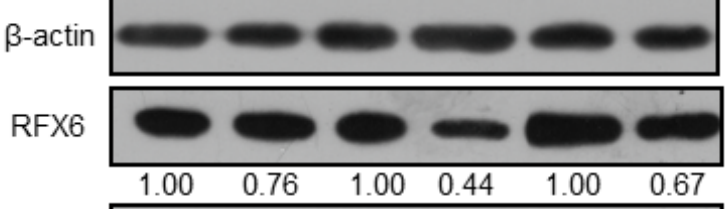

$\beta$-actin
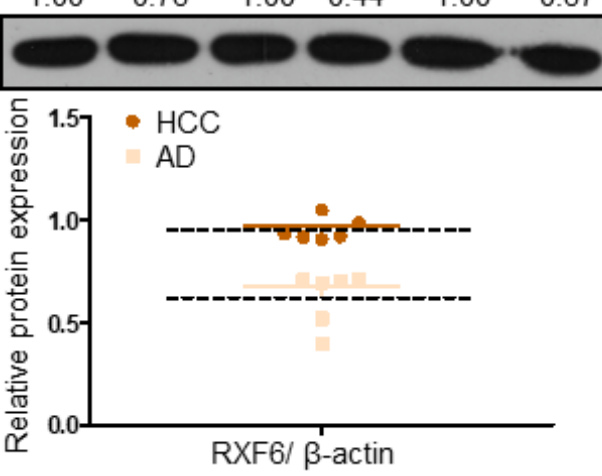

Figure 1

RFX6 highly expressed in hepatocellular carcinoma and negatively correlated with the prognosis. (a) TCGA data analysis of differently expressed genes in liver hepatocellular carcinoma tissues $(n=374)$ compared with adjacent normal tissues ( $n=50)$, (left panel). The paired comparison of RFX6 in TCGA database, containing human breast cancers $(n=50)$ and tumor adjacent normal tissues $(n=50)$, (right panel, ${ }^{*} p<0.05$ ). (b) Wilcox test was used to test the difference of RFX6 expression in different groups of clinical data to demonstrate the correlation between RFX6 expression and histologic grade. (c) Multivariate Cox regression analysis of patients based on the expression level of RFX6. (d) The protein levels of RFX 6 were higher in liver cancer tissue samples than in adjacent normal tissues, 6 pairs. 


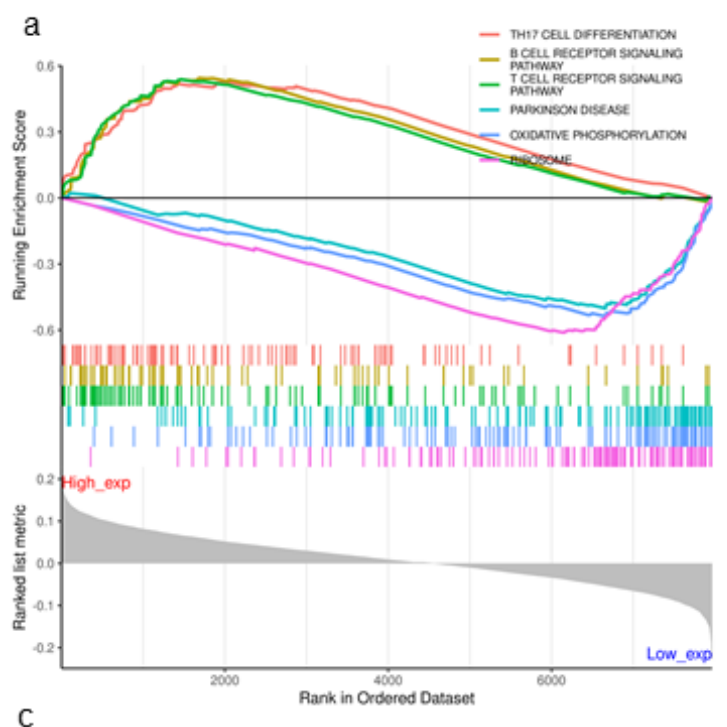

C
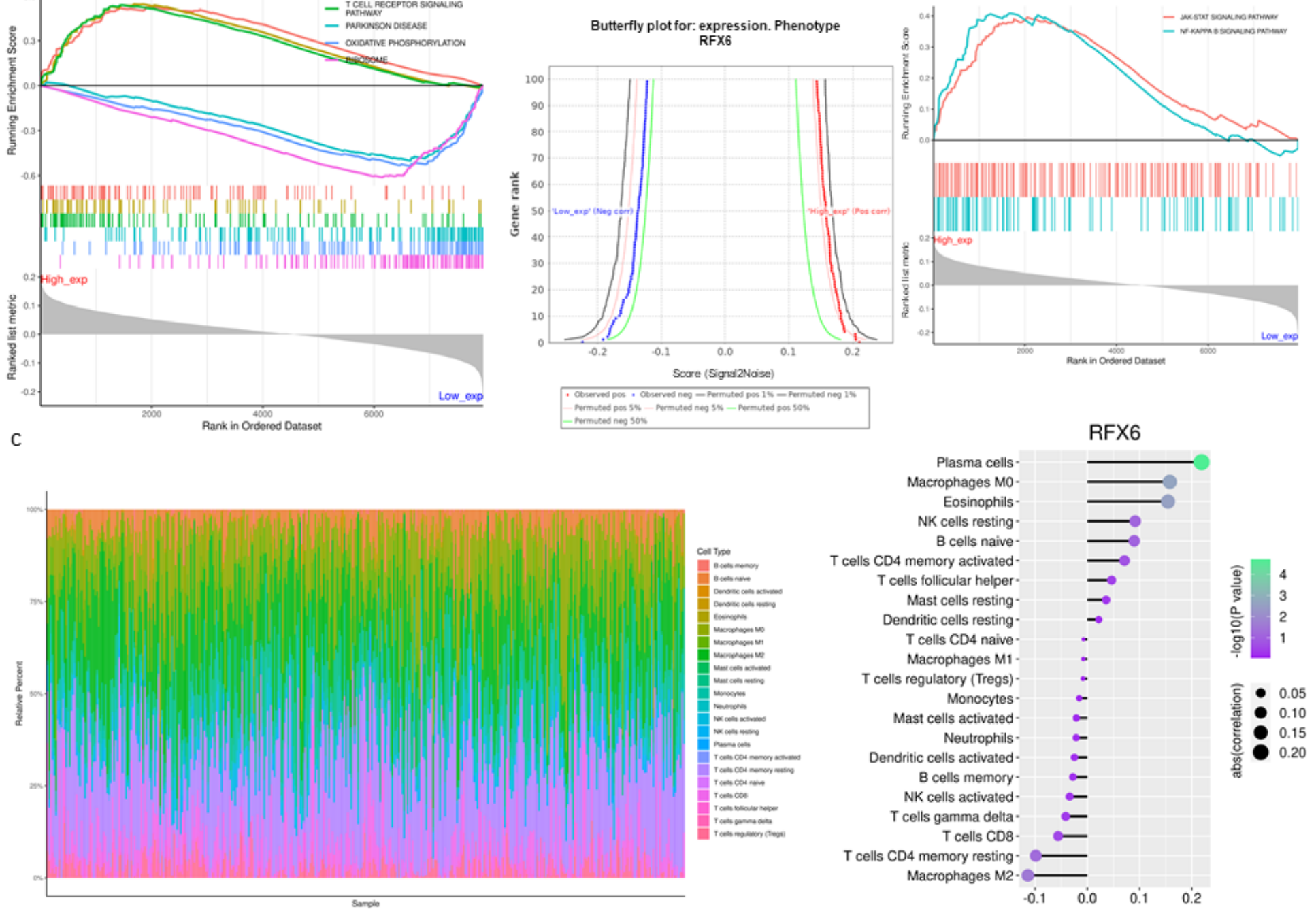

Figure 2

RFX6 affects immune cell infiltration in hepatocellular carcinoma microenvironment. (a) Gene Set Enrichment Analysis of KEGG pathways related with different RFX6 expression levels. (b) Butterfly plot for RFX6 expression phenotype (left panel). EMT pathways enrichment according to different RFX6 expression (right panel). (c) CIBERSORT barplot of different immune cell infiltration in hepatocellular carcinoma microenvironment (left panel). Point plot of immune cell correlation with RFX6 (right panel). 

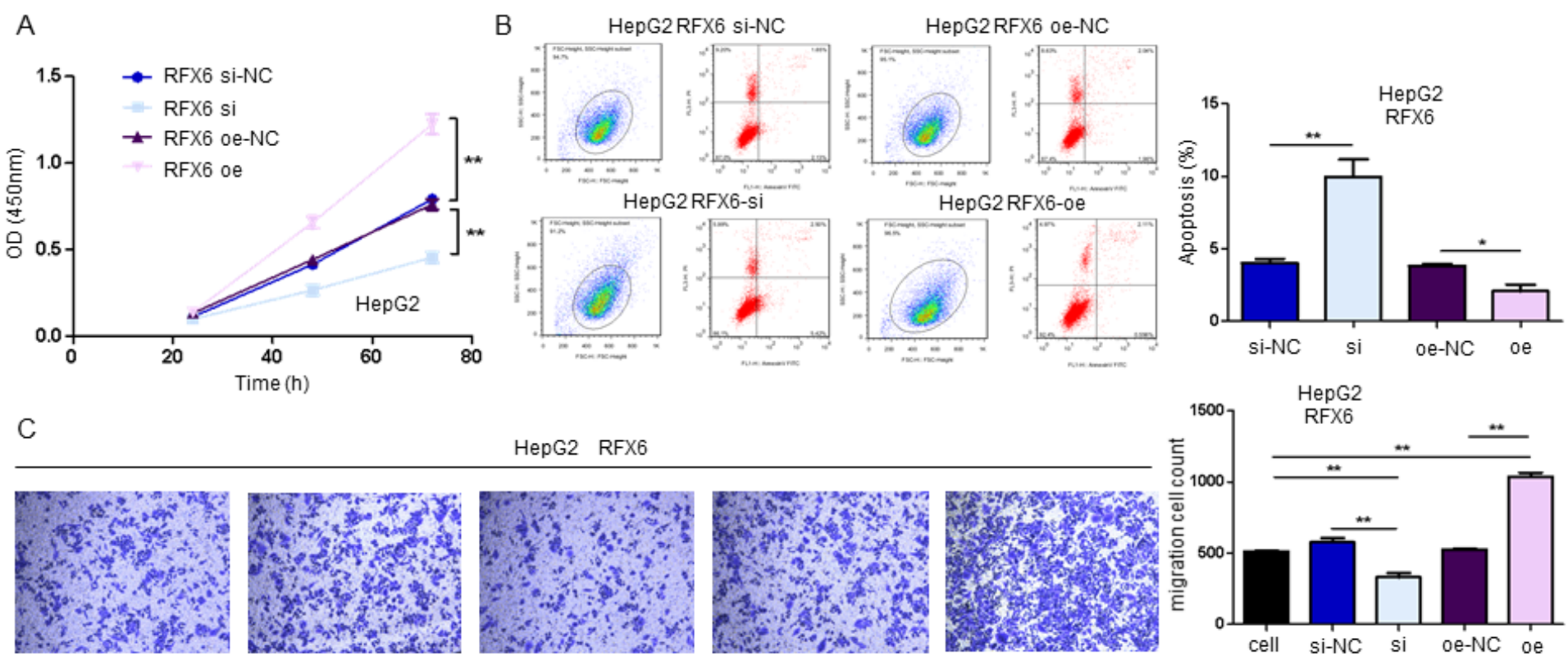

cell

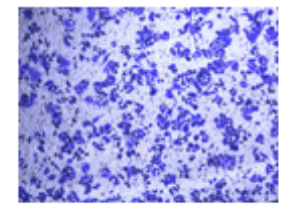

si-NC

HepG2 RFX6
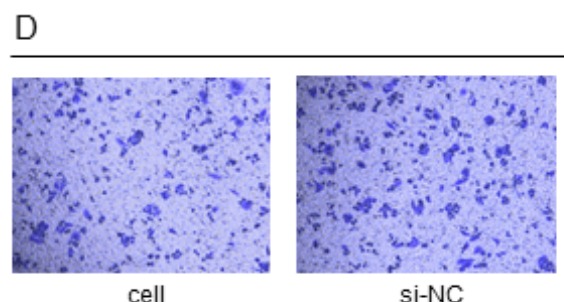

HepG2 RFX6
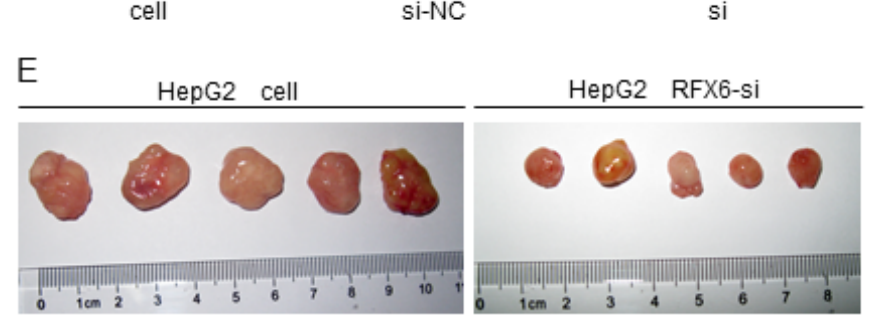

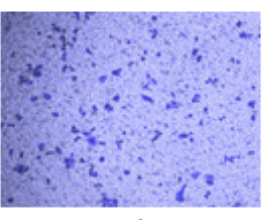

si

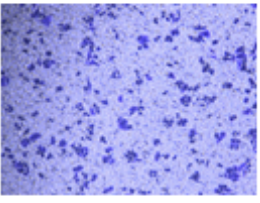

oe-NC

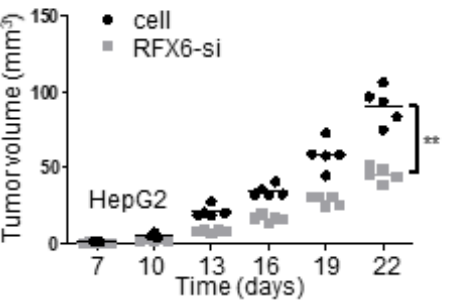

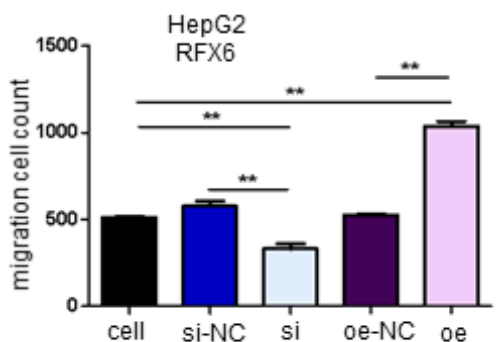

oe
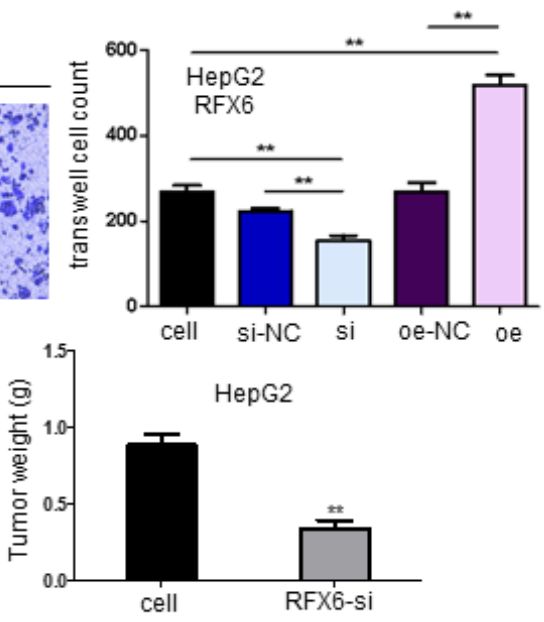

\section{Figure 3}

Expression of RFX6 promotes tumorigenesis of hepatocellular carcinoma. (a) The proliferation of HepG2 cells when altered the expression of RFX6 in cells. The cell number was determined with CCK-8 assay. Data were represented as mean \pm standard deviation (s.d.), $n=3$, $* \star p<0.01$. (b) The apoptosis of HepG2 before and after RFX6 alteration. Upper right (UR, PI+Annexin+) and Lower right (LR, PI-Annexin+) were counted as apoptotic cells. Data were represented as mean \pm standard deviation (s.d.), ${ }^{\star} p<0.05, * \star p$ $<0.01$. (c) Expression of RFX6 promoted the migration of HepG2 cells using a transwell assay. Data were represented as mean \pm standard deviation (s.d.), $n=3, * \star p<0.01$. (d) The knockdown of RFX6 could significantly inhibited the invasion of HepG2 cells. Data were represented as mean \pm standard deviation (s.d.), $n=3, * * p<0.01$. (e) The knockdown of RFX6 suppressed the tumor growth of HepG2 cells in nude mice, $n=5, * \star p<0.01$. 

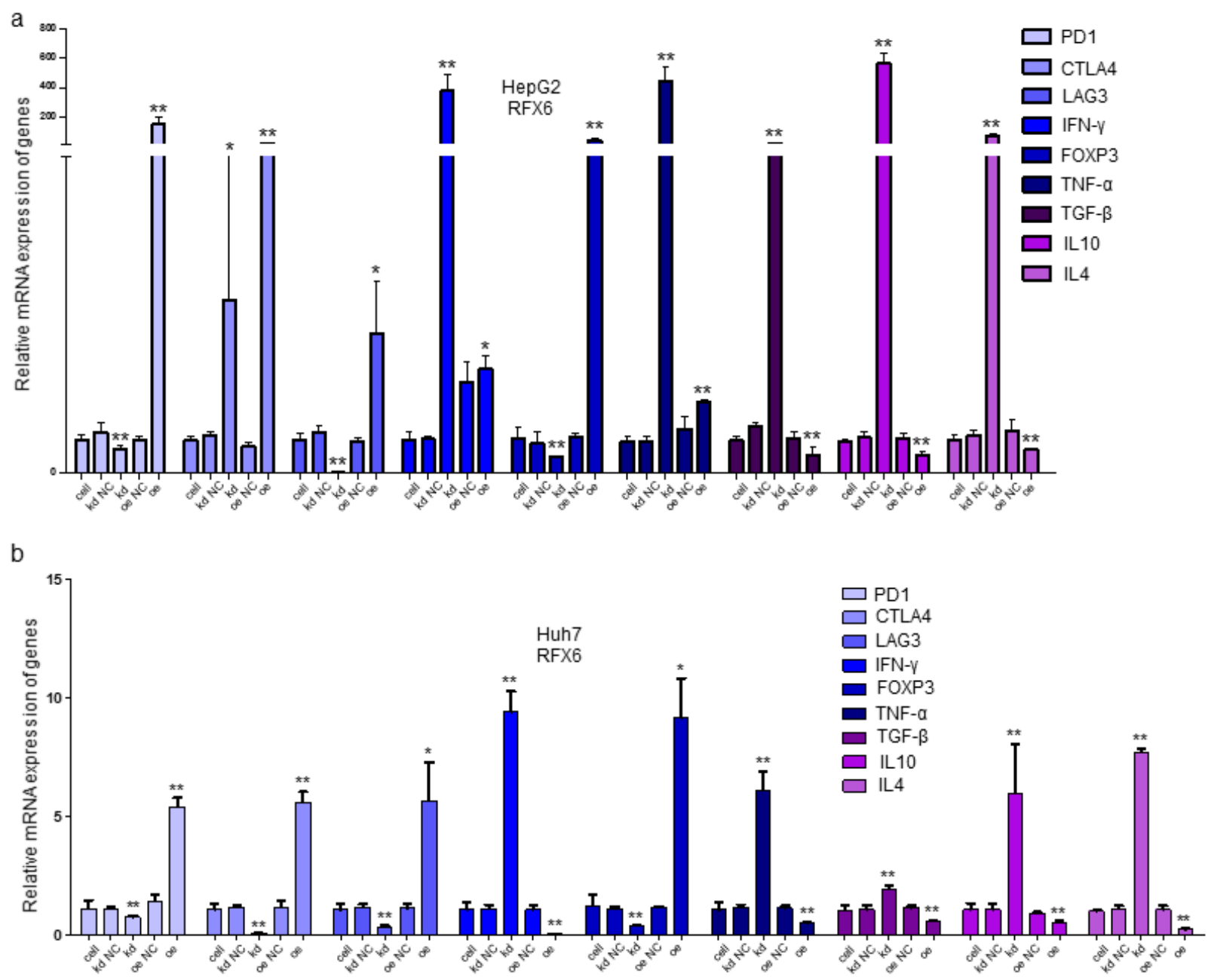

Figure 4

Significantly expressed marker genes of immune cells under different RFX6 expression levels. (a) mRNA expression changes of immune cell receptor marker genes in HepG2. Data were represented as mean \pm standard deviation (s.d.), $n=3,{ }^{*} p<0.05,{ }^{*} p<0.01$. (b) Relative expression of immune cell receptor mRNA in Huh7 after RFX6 alteration. Data were represented as mean \pm standard deviation (s.d.), $n=3, * p<0.05$, ** $p<0.01$. 


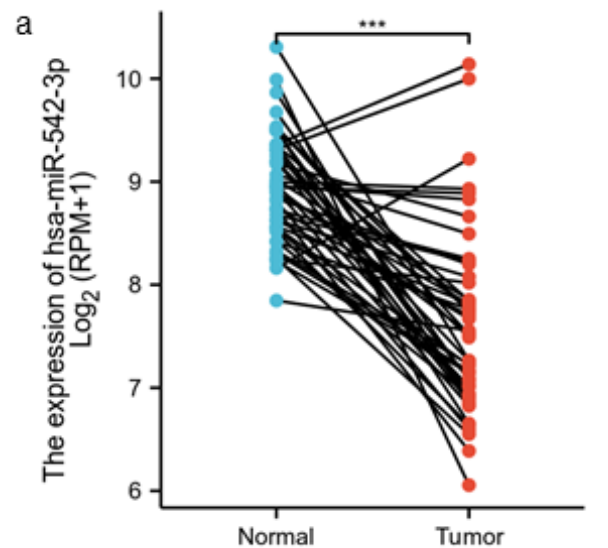

d

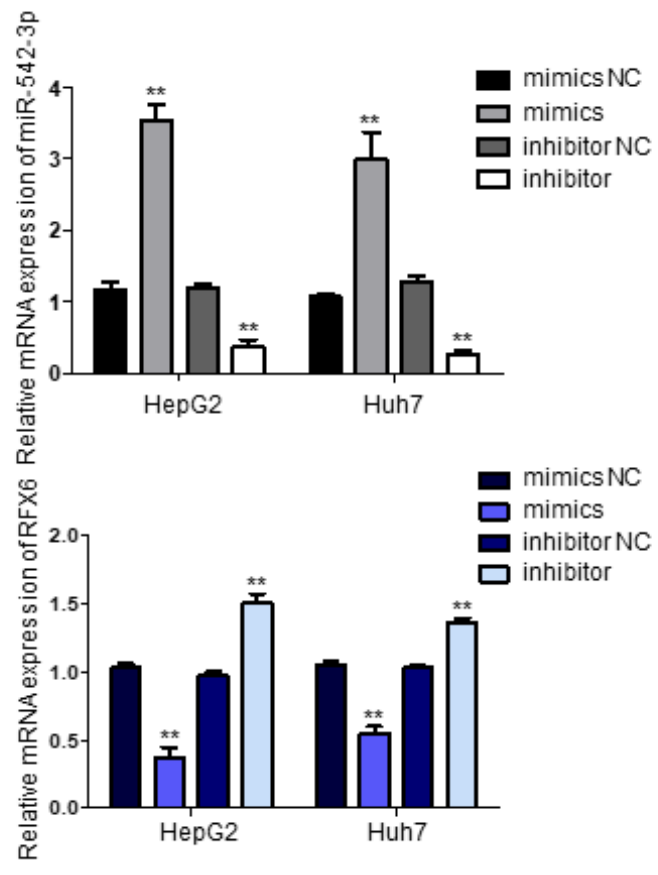

b

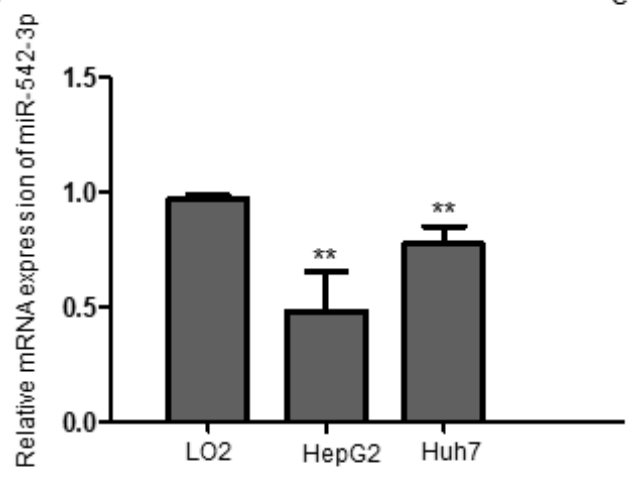

C

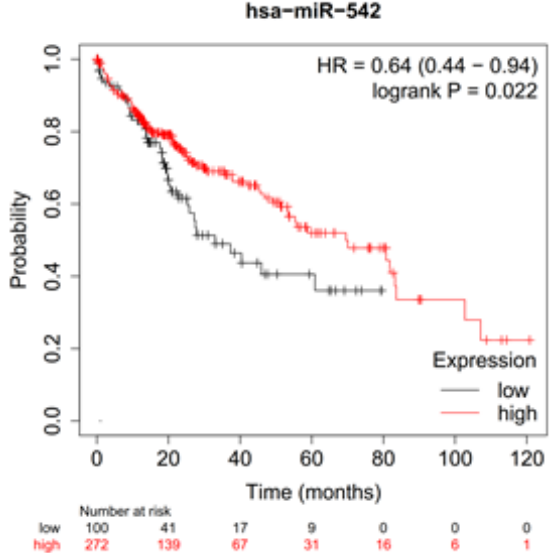

e

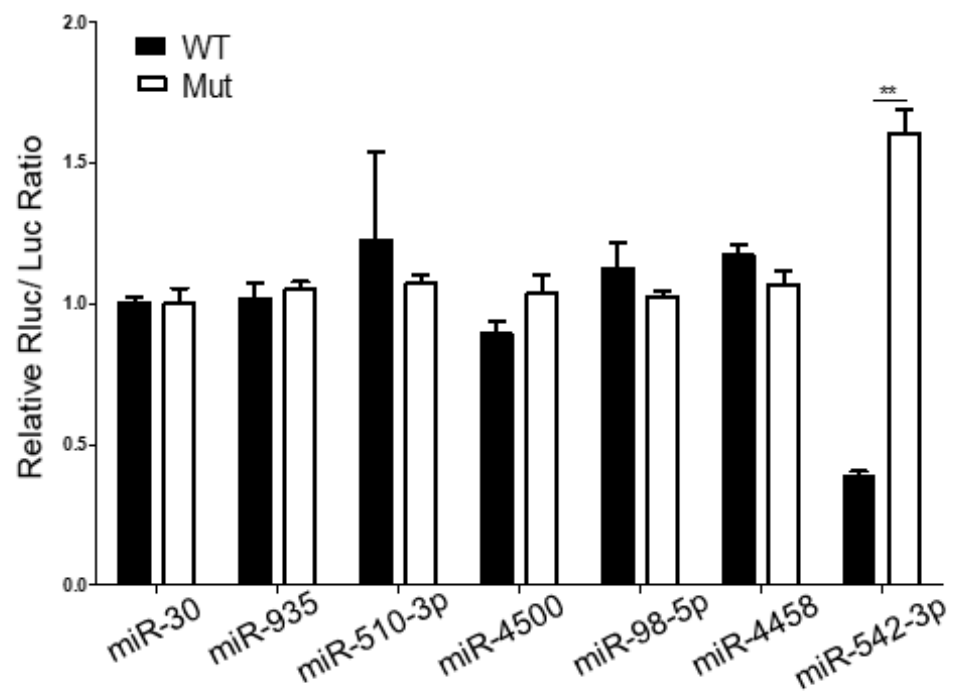

Figure 5

miRNA-542-3p negatively regulates RFX6 and positively correlates with the prognosis of human hepatocellular carcinoma. (a) The comparison of the expression level of miR-542-3p in human hepatocellular carcinoma $(n=374)$ and tumor adjacent normal tissues $(n=50)$ in TCGA database. (b) The expression of miR-542-3p was significantly reduced in liver cancer cells. Data were represented as mean \pm standard deviation (s.d.), $n=3, * \star p<0.01$. (c) Log-rank (Mantel Cox) survival test of hepatocellular carcinoma patients based on the levels of miR-542 (low expression $n=100$, high expression $n=272$ ), $p$ values were indicated. (d) Alteration of miR-542-3p expression inversely correlated with the levels of RFX6 mRNA. Data were represented as mean \pm standard deviation (s.d.), $n=3, \star \star p<0.01$. (e) miR-542-3p targeted with the predicted sites within the 3 -UTR of RFX6 and mutation of the predicted target sites rescued the inhibitory effects of RFX6. Data were represented as mean \pm standard deviation (s.d.), $n=3$, ** $\mathrm{p}<0.01$. 

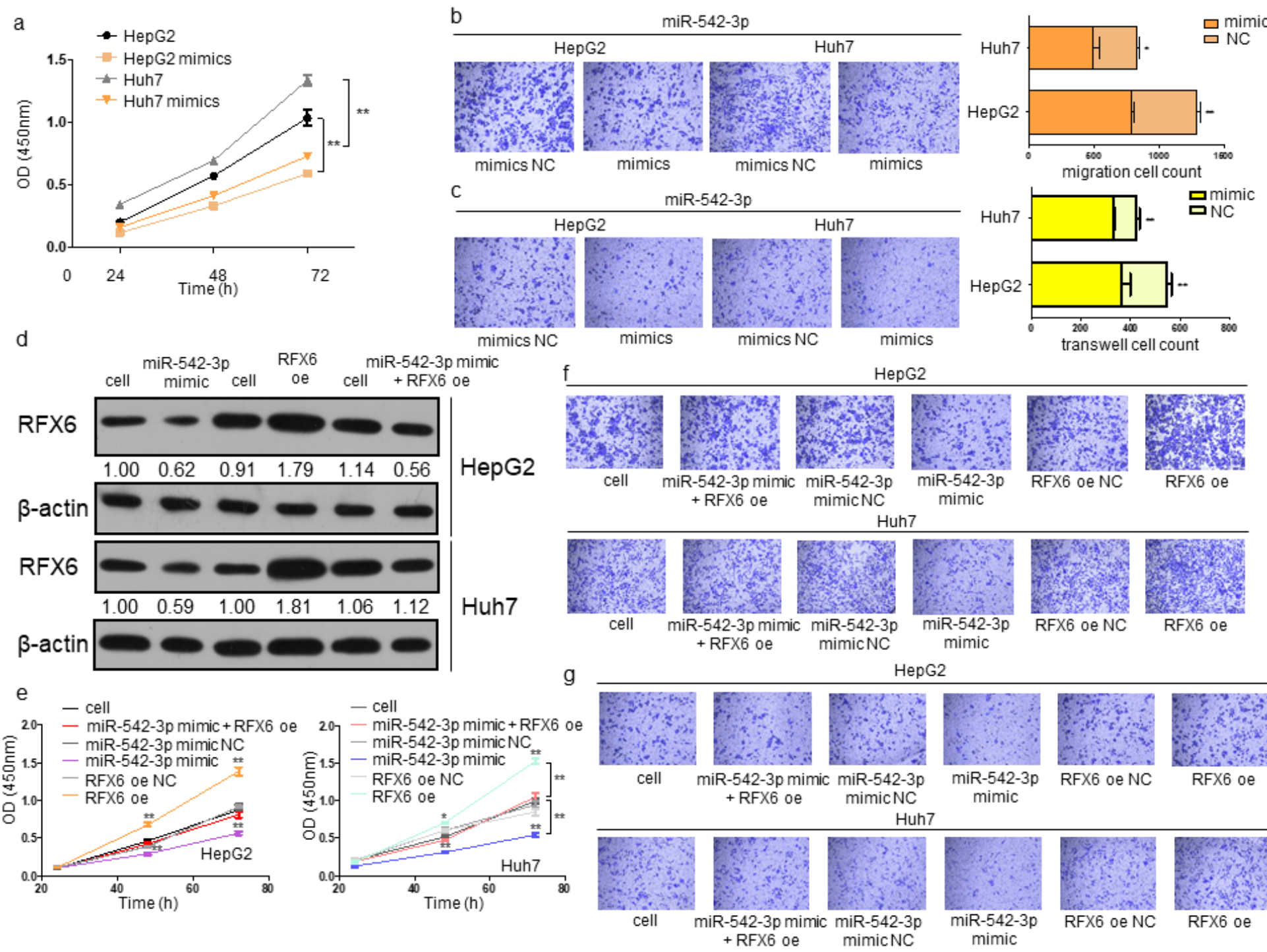

RFX6 oe

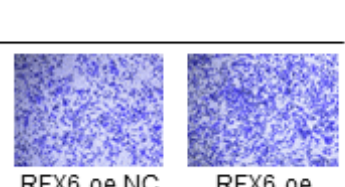

RFX6 oe NC

RFX6 oe
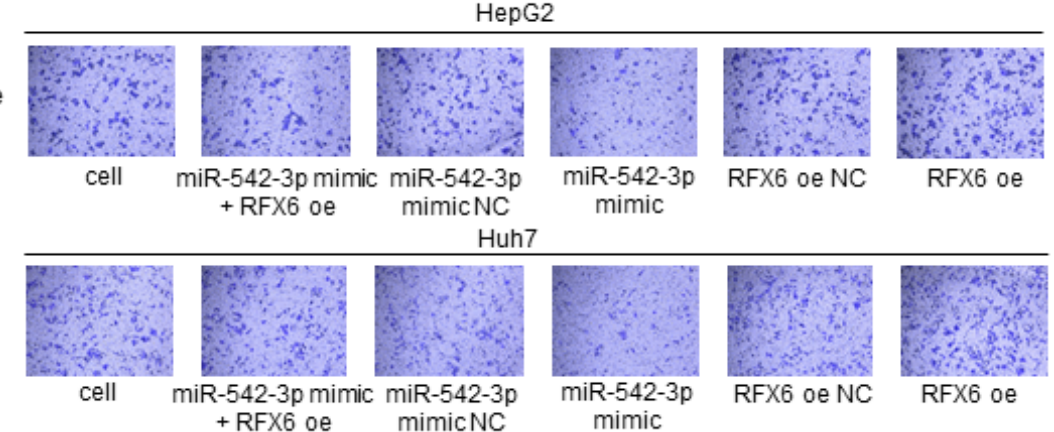

\section{Figure 6}

Downregulation of RFX6 by miRNA-542-3p affects invasion and oncogenesis of hepatocellular carcinoma cells. (a) Overexpression of miR-542-3p reduced the proliferation of liver cancer cells. The cell number was determined with CCK-8 assay. Data were represented as mean \pm standard deviation (s.d.), $n=3$, $* * p<0.01$. (b) miR-542-3p overexpression could significantly suppress the migration of HepG2 and Huh7 cells. Data were represented as mean \pm standard deviation (s.d.), $n=3, * \star p<0.01$. (c)

Overexpression of miR-542-3p reduced the invasion ability of liver cancer cells. Data were represented as mean \pm standard deviation (s.d.), $n=3, * \star p<0.01$. (d) Overexpression of RFX6 could reversed the protein level of RFX6 inhibited by miR-542-3p. (e) Proliferation of liver cancer cells when rescued the RFX6 expression. The cell number was determined with CCK-8 assay. Data were represented as mean \pm standard deviation (s.d.), $n=3, * \star p<0.01$. (f) Migration ability of liver cancer cells before and after rescued the expression of RFX6. (g) Rescue the expression of RFX6 affected the invasion ability of HepG2 and Huh7 cells. 

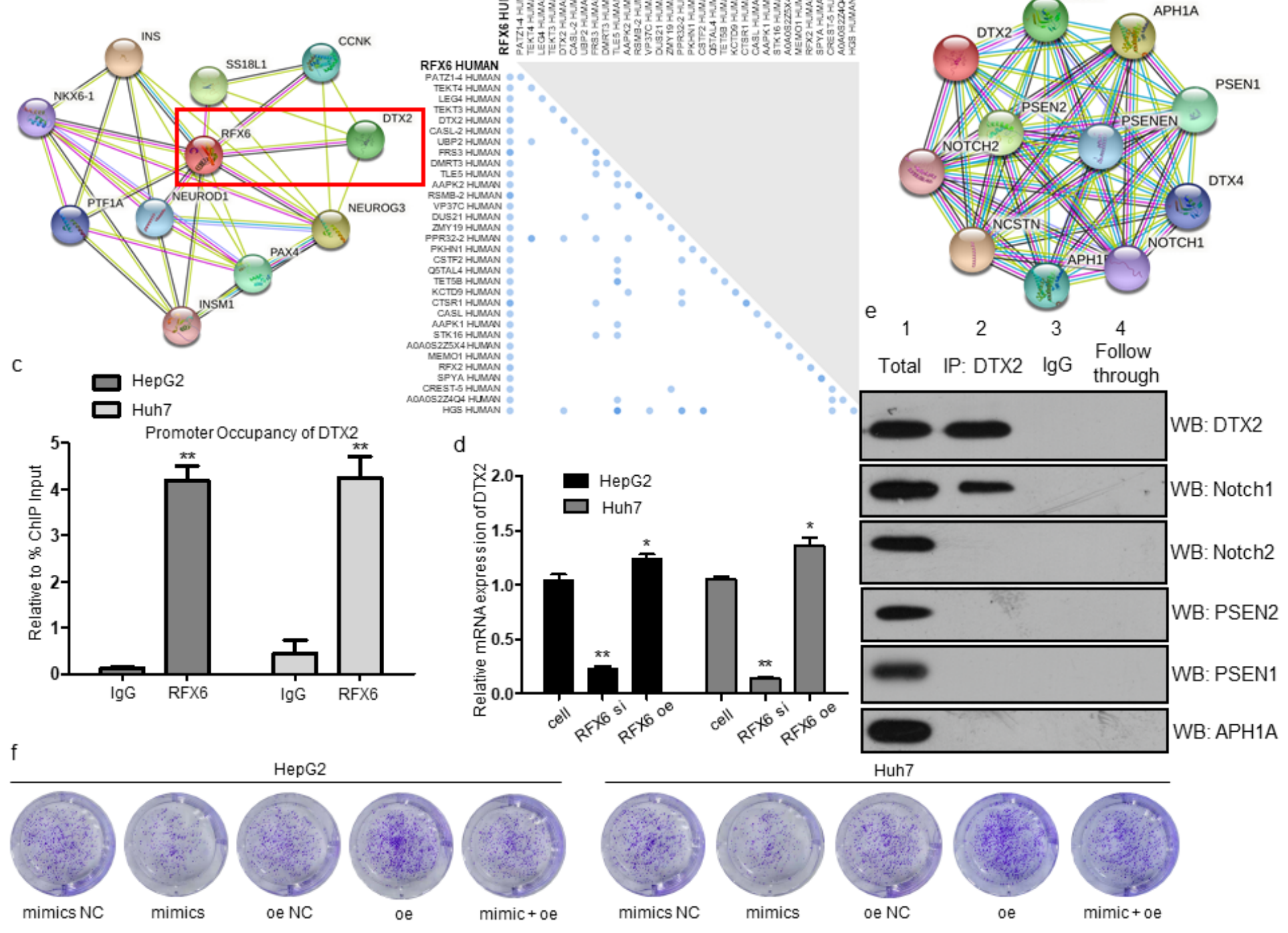

d

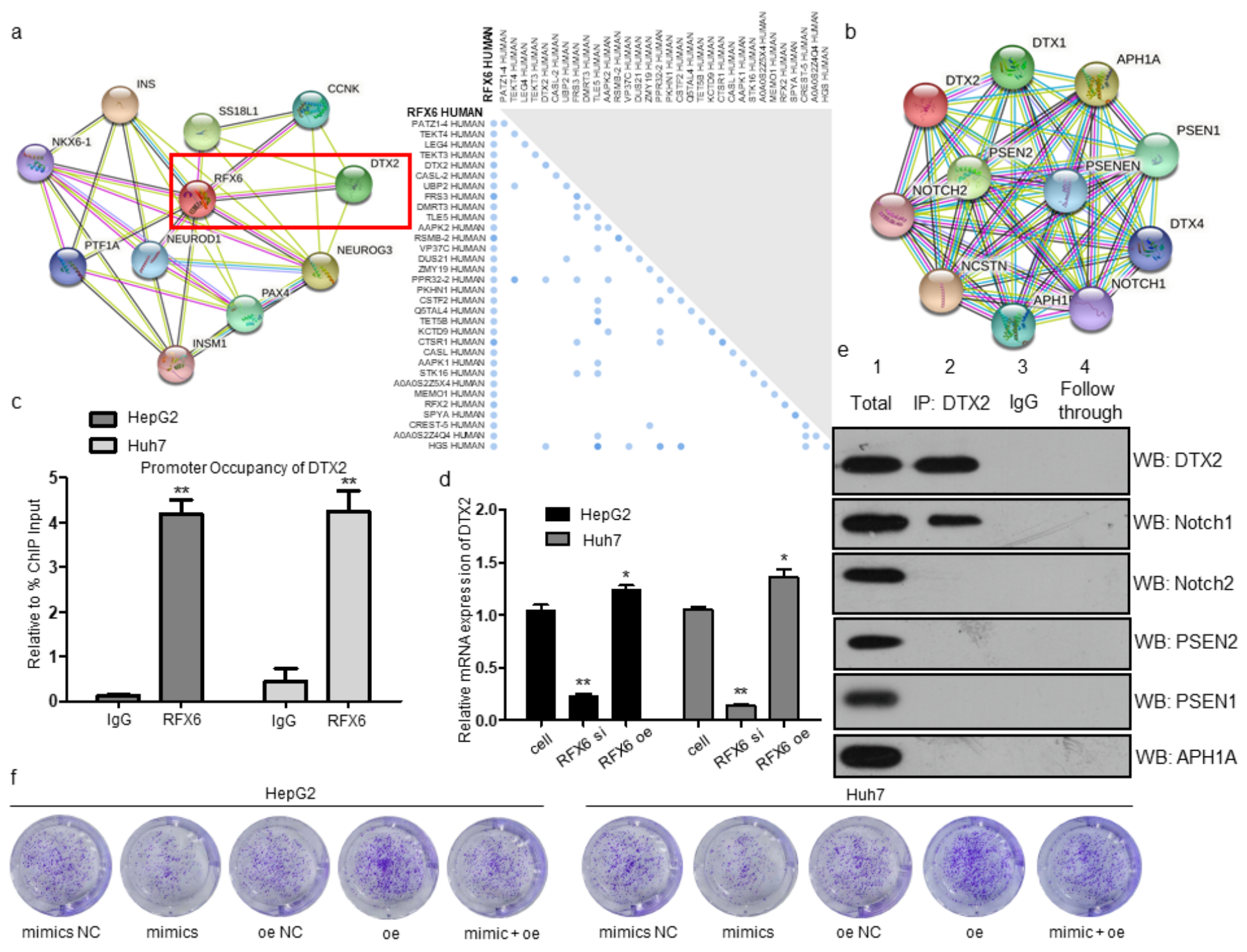

b

Figure 7

Expression of RFX6 promotes the translation of DTX2 and participates in Notch pathway. (a) STRING analysis predicted a network of proteins interacted with RFX6 (left panel). UniProt analysis of probable interacted proteins of RFX6 (right panel). (b) STRING analysis of possible interacting proteins of DTX2.

(c) Promoter occupancy of DTX2 gene by RFX6 performed by ChIP-qPCR. Data were represented as mean \pm standard deviation (s.d.), $n=3, * \star p<0.01$. (d) Expression of RFX6 increased the mRNA expression of DTX2. Data were represented as mean \pm standard deviation (s.d.), $n=3, * p<0.05$, ** $p<0.01$. (e) Notch1 was confirmed to interact with DTX2 in HepG2 cells by Co-IP assay. (f) Proliferation of liver cancer cells was retrained by downregulation of DTX2 conducted by miR-542-3p.

\section{Supplementary Files}

This is a list of supplementary files associated with this preprint. Click to download.

- Figures1.tif 
- Figures2.tif

- Figures3.tif 